\title{
Formación Básica en Higiene y Seguridad Laboral para Ingenieros
}

\author{
A.B. Sosa, L. Mattassini, L. Di Marco y R. Ferrari \\ Univ. Nacional de Tucumán, Facultad de Ciencias Exactas y Tecnología, Laboratorio de \\ Evaluación y Control de Contaminantes Ambientales (LECCA), Av. Independencia $N^{\circ} 1800$, \\ (4000) Tucumán -Argentina (e-mail: asosa@herrera.unt.edu.ar)
}

\begin{abstract}
Resumen
En este trabajo se propone la inclusión de la asignatura "Condiciones y Medio Ambiente de Trabajo. Higiene y Seguridad" en las carreras universitarias de Ingeniería en Argentina. La propuesta se basa en datos estadísticos de enfermedades profesionales y de accidentes, que en los años 1997 y 1998 alcanzaron en Argentina el 58.5\% en actividades relacionadas a la Ingeniería. La asignatura comprende el análisis de los factores de riesgos físicos, químicos, biológicos y síquicos, tratados en forma interdisciplinaria a través de clases teóricas. Las prácticas consistirían en determinaciones y evaluaciones de condiciones ambientales en plantas industriales y obras. La carga horaria propuesta es de seis horas por semana durante un cuatrimestre. Se concluye que es urgente la implementación de la propuesta, respondiendo al gran desafío de formar ingenieros comprometidos con la calidad de vida laboral.
\end{abstract}

Palabras claves: salud, seguridad e higiene, educación, ambiente de trabajo

\section{Basic Education in Hygiene and Occupational Safety for Engineers}

\begin{abstract}
This study proposes the inclusion of a Course Conditions and Working Environment. Hygiene and Safety in engineering university curricula in Argentina The proposal is based on statistical data on work-related illness and accidents, that in Argentina reached $58.5 \%$ of the work force engaged in engineering during 1997 and 1998. The course includes the analysis of physical, chemical, biological and psychological risk factors, given in interdisciplinary form through theoretical classes. Practical work would consist of determination and evaluation of environmental conditions in industrial plants and constructions. The hour load proposed would be six hours per week per academic quarter. It is concluded that the implementation of this proposal is urgent, as it responds to the challenge of forming engineers committed to the quality of working life.
\end{abstract}

Keywords: health, hygiene and safety, education, working environment

Nota: este artículo está tomado de "Información Tecnológica" [ISSN 0716-8756], vol. 11(4), 183-186 (2000) 


\section{INTRODUCCIÓN}

Si bien históricamente con anterioridad a 1900 el interés en la protección de la salud de los trabajadores era bajo, Clayton (1973) señala algunas observaciones realizadas en el pasado con relación a enfermedades laborales y algunos ejemplos son: Hipócrates (460-370 a.C) quién hizo referencias a tratamientos y enfermedades de accidentes laborales; Plinto, el viejo (22-79 d.C) que a comienzos de nuestra era hizo menciones similares y describió elementos de protección, como máscaras hechas con vejiga de animales; George Bauer, conocido como George Agrícola (1490 -1575) que en el siglo XVI, se ocupó de la ventilación de minas y señaló afecciones de mineros; Paracelso (1403-1541) quién es el autor de la obra "De los oficios y enfermedades de las montañas", donde hace referencia a la silicosis y las intoxicaciones de plomo y mercurio; Bemardino Ramazzini (1633-1714) médico italiano, a quién se considera el padre de la Salud Ocupacional, principalmente por su obra "De las enfermedades de los trabajadores", donde estudia y describe las enfermedades que afectaban a trabajadores de diferentes oficios.

El advenimiento de la revolución industrial dio origen a la organización industrial. Los campesinos, atraídos por el desarrollo de nuevas posibilidades laborales, migraron en masa hacia las grandes ciudades industriales, aumentando el número de trabajadores en fábricas y talleres. Todo esto sucedía en la ignorancia de los efectos que las maquinarias y los nuevos productos producían en la salud de los trabajadores. La historia muestra que el hombre muchas veces ha pagado y aún paga con su vida el derecho al trabajo. La Dou (1990), da una reseña de las principales enfermedades profesionales que se registran en los distintos tipos de trabajo. Las condiciones que rodean al hombre en su puesto de trabajo recibe el nombre de ambiente de trabajo. Las alteraciones y modificaciones del ambiente laboral son muy variadas, dependen de las actividades que allí se realicen.

La Organización Internacional del Trabajo (OIT, 1987) expresa que los riesgos profesionales a los que se halla sujeto el hombre tienen su origen en el mismo trabajo. Con los conocimientos actuales no se justifica el mantenimiento de condiciones hasta inhumanas de ambientes laborales existentes en muchos lugares del mundo. A esta situación se enfrentan muchos profesionales relacionados con la salud del trabajador, lo que queda patentizado como compromiso por De la Poza (1990), quién afirma "Nadie debe dudar, que actualmente, cuando todo el mundo está de acuerdo de que el hombre constituye el fundamento y fin de lo social, la preocupación por crear unas condiciones óptimas de trabajo se haya convertido en una de las exigen-ineludibles que debe poner en práctica cualquier sociedad moderna y civilizada.

La situación que se presenta en la República Argentina, en cuanto a los riesgos en puestos de trabajo, es una preocupación de organizaciones gubernamentales y no gubernamentales. Tales riesgos se traducen en hechos de daños personales, muchos de ellos con resultados fatales. La Superintendencia de Riesgos del Trabajo (1999), señala que en el término de un año (19971998), sobre un total de 82.788 siniestros registrados, el $58.5 \%$ correspondió a áreas relacionadas a la ingeniería, como ser "Explotación de minas y canteras", "Industrias Manufactureras", "Suministro de electricidad, gas y agua", "Construcción" y "Transporte, almacenamiento y comunicaciones". Estos valores, por si, justifican que el área de Higiene y Seguridad Industrial sea incorporada a la formación profesional del ingeniero en todas sus orientaciones. Pero, en general, no se observa que en las instituciones de formación de recursos humanos en la rama de ingeniería se le asigne la importancia dada al tema en otras profesiones, con las consiguientes pérdidas de posibilidades laborales. Además, la situación que presentan las principales industrias de la región en cuanto a los riesgos en puestos de trabajo, reafirma plenamente la necesidad de capacitación del futuro ingeniero en los temas que se proponen. Puesto que la eficacia en el desempeño de las tareas laborales depende fundamentalmente del estado físico y psíquico del individuo que las realiza, es de suma importancia brindar los medios necesarios para que pueda llevar a cabo su tarea en condiciones adecuadas. Esto significa que el futuro profesional debe estar capacitado para reconocer las condiciones de trabajo que atenten contra la salud del trabajador y que pueda discernir entre condiciones apropiadas y no apropiadas.

Tomando como base los puntos anteriores, el objetivo es proponer a las autoridades universitarias la inclusión, con carácter obligatorio, de la temática de las Condiciones y Medio 
Ambiente de Trabajo en la currícula de formación profesional del Ingeniero, a través de la materia "Condiciones y Medio Ambiente de Trabajo, Higiene y Seguridad", acción dirigida a lograr que los alumnos:

i) Analicen los fundamentos teóricos de las Condiciones y Medio Ambiente de Trabajo.

ii) Reflexionen sobre la responsabilidad del ingeniero en la Prevención de Accidentes y Cuidados del Medio Ambiente.

iii) Se capaciten en el Manejo de Instrumentos de Medición y Cálculos Básicos relacionados con el Medio Ambiente Laboral.

iv) Propongan cambios que permitan el desarrollo de las actividades laborales con mínimos riesgos.

\section{CONTENIDOS DE LA ASIGNATURA}

Los conceptos que se tratan en la asignatura, tales como ruido, radiaciones ionizantes y no ionizantes, agentes asfixiantes, entre otros, están presentes en mayor o menor grado en cualquier actividad Industrial. Durante el desarrollo de la materia, el alumno recibirá información general sobre los conceptos de riesgos laborales, contaminantes físicos y químicos y sus efectos sobre la salud, seguridad, las medidas de prevención y elementos de protección personal. Con esto se pretende lograr una capacitación del futuro profesional que lo familiarice con las condiciones de trabajo que encontrará en su vida laboral, prepararlo para el trabajo en condiciones adecuadas y al mismo tiempo para que sea generador de empleos satisfactorios. Con este objetivo los alumnos realizarán trabajos prácticos de determinaciones en el laboratorio, muestreos en industrias de la zona y visitas a laboratorios que cuentan con equipos de afta tecnología. En lo que se refiere a contaminantes que se originan en el ambiente de trabajo y que pueden aparecer dentro y fuera del mismo, se hace hincapié en cálculos para cuantificar sus magnitudes y en los elementos de protección apropiados para cada uno de ellos. Se consideran relevantes las nociones sobre leyes y Normas en Higiene y Seguridad y Estándares de Niveles de Contaminantes Máximos Permisibles, las cuales permiten prevenir los accidentes y arbitrar los medios legales necesarios para tal fin. Tratamiento aparte merece el tema de Organización del Trabajo, donde se incentiva al alumno al trabajo en equipos multidisciplinarios a fin de encontrar soluciones a los problemas organizacionales tales como identificación con la tarea, motivación, liderazgo, distribución y duración de las jornadas de trabajo, carga mental de trabajo, automatización y comunicación. Para el tratamiento sistemático de la asignatura, ésta se divide en los temas mostrados en la Tabla 1.

Tabla 1: Temas a tratar en la asignatura propuesta "Condiciones y Medio Ambiente de Trabajo, Higiene y Seguridad"

\footnotetext{
Primer Grupo de factores de riesgos

- lluminación.

- Temperatura.

- Carga térmica.

- Ventilación - humedad.

- Radiaciones.

- Ruidos - vibraciones.

Segundo grupo de factores de riesgos

- Contaminantes químicos.

- Contaminantes biológicos.

Tercer grupo de factores de riesgos

- La carga del trabajo.

- Posturas de trabajo.

- Ergonomía.
}

Cuarto grupo de factores de riesgos

- Organización del trabajo.

- Jornadas de trabajo.

- Trabajo nocturno y rotativo.

- Automatización y monotonía.

- Crisis socioeconómica.

- Participación.

Quinto grupo de factores de riesgos

- Las condiciones de seguridad.

- identificación de las fuentes de riesgos.

- Inspección de Seguridad. Señalización.

- Protección personal.

Leyes y Normas

- Estándares de niveles máximos permisibles.

Prácticas en industrias

Prueba de evaluación final 


\section{PROCEDIMIENTO}

La forma de implementar la asignatura involucra recursos, metodología y evaluación, aspectos presentados en lo que sigue:

Recursos: Los recursos empleados para llevar a cabo el desarrollo de asignatura están divididas en: i) Para las clases teóricas: Medios audiovisuales: Videos, filminas; Material bibliográfico, Revistas, libros. Internet; Participación en el desarrollo de los temas: Docentes. Profesionales y técnicos de empresas; y ii) para las clases prácticas: Instrumental para medición de ruidos, iluminación, gases, vapores y partículas en ambientes de trabajo, en especial portátiles y aceptados por las normas vigentes.

Metodología: La asignatura se desarrollará en un cuatrimestre en 6 horas semanales, distribuidas en clases teóricas y prácticas, con una evaluación final. La asignatura se ubica en el cuarto año de formación básica profesional de las carreras de Ingeniería. i) Clases teóricas: Desarrolladas por Docentes, Profesionales responsables de Higiene y Seguridad de empresas e invitados al efectoy ii) Clases prácticas: Calibración de equipos y Evaluación de contaminantes en ambientes de trabajo en empresas del medio (mapeo de riesgos e informes).

Evaluación: Para la promoción de la asignatura los alumnos deberán cumplir con actividades prácticas en empresas de la zona. Esta tarea podrá realizarse en forma grupal y consistirá en la medición de contaminantes en ambientes de puestos de trabajo y en la realización de un mapa de riesgos. A partir de los valores obtenidos, harán una evaluación de la situación existente y propondrán cambios que consideren necesarios para mejorar la calidad del ambiente laboral en estudio. Esta propuesta debe ser defendida ante sus compañeros y el tribunal. Se considera conveniente la participación del encargado de Higiene y Seguridad de la empresa en la cual se realizó el estudio.

\section{DISCUSIÓN}

En el ámbito de una empresa, sea de producción o de servicio, se presentan situaciones de riesgo que deben ser analizadas para eliminar o al menos minimizar los efectos adversos para la salud del personal. El origen de muchas enfermedades laborales se debe al uso de tecnologías no apropiadas y al desconocimiento de la toxicidad de los productos empleados, siendo por lo tanto muy importante la formación del ingeniero en ese campo. En tal sentido se ha expresado el Consejo Federal de Decanos de Ingeniería (CONFEDI, 1996) en las recomendaciones de "Unificación Curricular en la Enseñanza de las Ingenierías en la República Argentina".

Si bien la temática de Higiene y Seguridad es tratada en cursos de postgrado, se considera conveniente que todo estudiante de ingeniería reciba una formación en los temas de prevención de accidentes y de los factores de riesgos presente en ambientes laborales. Esto se lograría a través de la inclusión de una asignatura en los planes de estudios de grado en las distintas especialidades de Ingeniería, lo que permitiría también descubrir la vocación de muchos jóvenes hacia la prevención de riesgos y despertar en ellos el Interés hada el mejoramiento de la calidad de vida en las distintas situaciones de trabajo.

La tarea en este sentido será en muchos casos muy ardua, sobre todo en convencer a miembros de las entidades educacionales que consideran que el campo es muy específico y se resisten al tratamiento del tema bajo un enfoque multidisciplinario La participación de psicólogos laborales, higienistas, químicos, abogados, es esencial para encontrar soluciones adecuadas a las complejas situaciones que surgen en los distintos tipos de tareas. No debemos olvidar que el trabajo es el motor de las sociedades, y que eleva el nivel de vida, promoviendo el equilibrio físico, mental y social de las comunidades que las malas condiciones de trabajo pueden destruir. En este nuevo siglo el gran desafío del cambio está en la educación del hombre en el cuidado de su prójimo y de su hábitat y las universidades no deben mantenerse ajenas a ese desafío.

\section{CONCLUSIONES}

Se considera que la implementación de la asignatura propuesta se justifica plenamente, ya que atender un punto tan importante como es la prevención de accidentes y enfermedades laborales es un objetivo que hoy en día es exigido por la sociedad y por parámetros de calidad establecidos por 
normas. Las universidades, en especial en las carreras de ingeniería, no pueden eludir la responsabilidad de atender la formación de recursos humanos que cuenten con los conocimientos necesarios para identificar y disminuir los riesgos originados en actividades laborales.

\section{REFERENCIAS}

Clayton G.O.; U.S. The Industrial Environment - its Evaluation and Control, Department of health and human services, U.S. Goverment Printing Office, Washington, D.C. 20402: (1), 1-5 (1973).

CONFEDI; Consejo Federal de Decanos de Ingeniería, Unificación Curricular en la Enseñanza de las Ingenierías en la República Argentina, Proyecto ICI-CONFEDI (1996).

De la Poza, J.M.; Seguridad e Higiene Profesional, Paraninfo: (1), 29 (1990)

La Dou, J.; Medicina Laboral Manual Moderno México, D.F. :(1). 1-5 (1990).

OIT, Oficina Internacional del Trabajo; Introducción a las Condiciones y el Medio Ambiente del Trabajo, Ginebra: (2), 45 (1987).

Superintendencia de Riesgos de Trabajo; Internet: http://www. srt.gov.ar (1999) 